\title{
Update on tuberculosis and other opportunistic infections associated with drugs blocking tumour necrosis factor $\alpha$
}

\section{K L Winthrop}

$\mathrm{T}$ he World Health Organization estimates that a third of the world's population is infected with tuberculosis (TB). In approximately $90 \%$ of people, the infection remains latent. In others, TB disease develops, often in the first two years after infection. Individuals whose immune systems are compromised by disease or drugs are at increased risk for developing TB disease.

The pathogenesis of $\mathrm{TB}$ is complex. In pulmonary $\mathrm{TB}$, inhaled TB bacilli are first engulfed by alveolar macrophages. Next, they replicate and briefly disseminate haematogenously throughout the body. The host attempts to limit bacilli spread with granuloma formation, a process mediated by tumour necrosis factor (TNF) $\alpha .{ }^{12}$ This proinflammatory cytokine is released by activated immune cells. It mediates systemic inflammatory responses and tissue destruction, and is important in the pathogenesis of autoimmune diseases such as rheumatoid arthritis, psoriasis, and Crohn's disease.

Recently, TNF $\alpha$ blocking drugs (infliximab, etanercept, and adalimumab) have been shown to be highly successful in the treatment of the above mentioned autoimmune diseases and other conditions. However, infectious complications have been reported with all three drugs including the reactivation of latent TB infection, which often presents atypically in disseminated or extrapulmonary fashion. ${ }^{3-7}$ Other infections have also been reported including non-tuberculous mycobacteriosis, histoplasmosis, listeriosis, aspergillosis, nocardiosis, and others. ${ }^{6}$

The US Food and Drug Administration tracks voluntary reports of such complications in their adverse events database. The most recent review of this database (cases reported from January 1998 through September 2002) found 335 cases of infliximab associated TB and 39 cases of etanercept associated TB worldwide. The cumulative incidence of reported TB among patients in the USA receiving these drugs during the time period studied was estimated at $54 / 100000$ for infliximab and 28/100 000 for etanercept. The authors found that onset after drug administration was sooner in the cases associated with infliximab than with etanercept. ${ }^{6}$ Their findings suggest that infliximab carries a higher risk of TB than does etanercept. Although differences in biological mechanisms between the two drugs have been proposed to explain this observed risk difference, ${ }^{28}$ it is also possible that underlying differences in the populations that received the two drugs might have contributed to this finding. Differences in underlying risk factors for $\mathrm{TB}$, or in the use of concomitant methotrexate or corticosteroids, could confound the analysis of this apparent risk difference. Further studies comparing the risk of TB between these drugs need to control for such potential differences in the two populations receiving the drugs.

Any patient who will receive TNF $\alpha$ blocking drugs should be screened for latent TB infection before treatment. A careful medical history should be taken assessing the patient for risk factors of TB, including history of birth or extended residence in a country with high TB prevalence, previous contact with a case of TB, previous history of latent TB infection or treatment, history of incarceration or homelessness, or intravenous drug use. All patients should receive a tuberculin skin test (TST). Patients who are already immunosuppressed by either disease or medication are more likely to have false negative TST results. For such people, a $5 \mathrm{~mm}$ induration should be considered positive, and for those with risk factors for TB, a negative TST should be interpreted with caution. ${ }^{5}$ Anergy panel testing is not recommended to assist with the interpretation of a negative TST result, as previous studies have failed to demonstrate its usefulness in human immunodeficiency virus (HIV)-infected individuals. ${ }^{9}$ Alternatives to the TST include new, blood based assays that assess interferon $-\gamma$ response to TB antigens that are highly specific for TB and not found in the Bacillus CalmetteGuérin (BCG) vaccine. These tests appear to offer a more specific way to detect latent TB infection; however, experience in screening immunosuppressed populations with these assays has been limited to date. ${ }^{10}$

In all individuals with a positive screening test, or in individuals where epidemiological suspicion is high for latent TB infection despite a negative screening test, a chest radio graph should be taken to rule out active TB disease. Patients diagnosed as having latent TB infection should begin preventive treatment before starting TNF $\alpha$ blocking drugs. Currently, nine months of isoniazid (INH) is the preferred therapy, except in instances where patients have been exposed to INH resistant cases of TB. Four month therapy with rifampicin alone, or in combination with INH, is a recommended alternative. ${ }^{11}{ }^{12}$ Although clinically significant INH hepatotoxicity is rare, ${ }^{13}$ individuals with underlying liver disease or those taking other hepatotoxic medications, should have their hepatic function monitored regularly during INH therapy. ${ }^{11}$

In patients who develop TB disease while on TNF $\alpha$ blocking drugs, anti-TNF therapy should be stopped immediately and standard four drug therapy for TB instituted. While treatment for TB has been successful in persons using other immunosuppressants (that is, corticosteroids, azathioprine, and ciclosporin), ${ }^{14}$ few data exist regarding concomitant use of anti-TNF $\alpha$ drugs and TB therapy in TB patients. One small study $(n=16)$ found the concurrent use of etanercept to be safe in HIV-infected individuals being treated for active TB disease. ${ }^{15}$ Further study of this question is warranted.

The US Centers for Disease Control and Prevention has recently published interim screening recommendations for this patient population, ${ }^{5}$ and is in the process of convening clinical experts in rheumatology and other specialty fields to review current research and formulate refined TB screening and treatment guidelines. Further research should seek to clarify the potential TB risk difference between different TNF $\alpha$ blocking drugs, and to better understand the impact of such drugs on the treatment of TB.

Competing interests: none declared

Correspondence to: Kevin L Winthrop, Good Samaritan Hospital Clinic, 1200 NW 23rd Avenue, Portland, Oregon USA 97210; kwinthro@lhs.org 


\section{REFERENCES}

1 Tufariello JM, Chan J, Flynn JL. Latent tuberculosis: mechanisms of host and bacillus that contribute to persistent infection. Lancet Infect Dis 2003:3:578-90.

2 Gardam MA, Keystone EC, Menzies R, et al. Anti-tumour necrosis factor agents and tuberculosis risk: mechanisms of action and clinical management. Lancet Infect Dis 2003;3:148-55.

3 Keane J, Gershon S, Wise RP, et al. Tuberculosis associated with infliximab, a tumor necrosis factor $-\alpha$ neutralizing agent. N Engl J Med 2001;345:1098-104.

4 Mohan AK, Cote TR, Block JA, et al. Tuberculosis following the use of etanercept, a tumor necrosis factor inhibitor. Clin Infect Dis 2004;39:295-9.

5 Centers for Disease Control, Prevention (CD)C). Tuberculosis associated with blocking agents against tumor necrosis factor-alpha, California 2002-2003. MMWR Morb Mortal Wkly Rep 2004;53:683-6.

6 Wallis RS, Broder MS, Wong JY, Hanson ME, Beenhouwer DO Granulomatous infectious diseases associated with tumor necrosis factor antagonists. Clin Infect Dis 2004;38:1261-5; correction Clin Infect Dis 2004;39:1254-5.

7 Ellerin T, Rubin RH, Weinblatt ME. Infections and anti-tumor necrosis factor alpha therapy. Arthritis Rheum 2003;48:3013-22

8 Arend SM, Breedveld FC, Dissel JT van. TNF- $\alpha$ blockade and tuberculosis: better look before you leap. Neth J Med 2003;61:111-19.
9 Anergy Skin Testing and Preventive Therapy for HIV-Infected Persons: Revised Recommendations. Centers for Disease Control and Prevention. MMWR Recomm Rep, 1997;46(No. RR-15):1-19.

10 Pai M, Riley LW, Colford JM Jr. Interferon-gamma assays in the immunodiagnosis of tuberculosis: a systematic review. Lancet Infect Dis 2004;4:761-76.

11 American Thoracic Society. Targeted tuberculin testing and treatment of latent tuberculosis infection. Am J Respir Crit Care Med 2000;161:S221-S247.

12 Centers for Disease Control and Prevention and Prevention (CDC); American Thoracic Society. Update: adverse event data and revised American Thoracic Society/CDC recommendations against the use of rifampin and pyrazinamide for treatment of latent tuberculosis infection-United States, 2003. MMWR Morb Mortal Wkly Rep, 2003;52:735-9.

13 Nolan C, Goldberg SV, Buskin SE. Hepatotoxicity associated with isoniazid preventive therapy: a 7 year survey from a public health tuberculosis clinic. JAMA 1999;281:1014-18.

14 Singh N, Paterson DL. Mycobacterium tuberculosis. infection in solid-organ transplant recipients: impact and implications for management, Clin Infect Dis 1998;27:1266-77.

15 Wallis RS, Kyambadde P, Johnson JL, et al. A study of the safety, immunology, virology, and microbiology of adjunctive etanercept in HIV-1-associated tuberculosis. AIDS 2004; 18:257-64. 